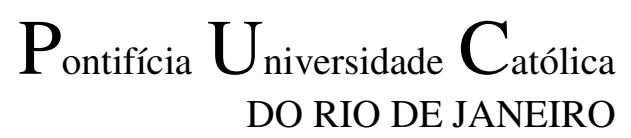

DO RIO DE JANEIRO

Eduardo Maria Terra Morelli

\title{
Recriação Automática de Índices em um SGBD Relacional
}

\section{Dissertação de Mestrado}

Dissertação apresentada ao Programa de Pósgraduação em Informática da PUC-Rio como requisito parcial para obtenção do grau de Mestre em Informática.

Orientador: Prof. Sérgio Lifschitz

Rio de Janeiro, setembro de 2006 


$$
\begin{array}{r}
\text { Pontifícia }_{\text {Do RIO DE JANEIRO }} \\
\mathrm{U}_{\text {niversidade }} \mathrm{C}_{\text {atólica }}
\end{array}
$$

Eduardo Maria Terra Morelli

\title{
Recriação Automática de Índices em um SGBD Relacional
}

\begin{abstract}
Dissertação apresentada como requisito parcial para obtenção do título de Mestre pelo Programa de PósGraduação em Mestrado em Informática do Departamento de Informática do Centro Técnico e Científico da PUC-Rio. Aprovada pela Comissão Examinadora abaixo assinada.
\end{abstract}

Prof. Sérgio Lifschitz Orientador PUC-Rio

Prof. Rubens Nascimento Melo Departamento de Informática - PUC-Rio

Prof. Renato Fontoura de Gusmão Cerqueira Departamento de Informática - PUC-Rio

Prof. Geraldo Zimbrão da Silva Departamento da Ciência da Computação - UFRJ

Prof. Asterio Kiyoshi Tanaka Departamento de Informática Aplicada - UNIRIO

Prof. José Eugenio Leal Coordenador(a) Setorial do Centro Técnico Científico - PUC-Rio 
Todos os direitos reservados. É proibida a reprodução total ou parcial do trabalho sem autorização da universidade, do autor e do orientador.

\section{Eduardo Maria Terra Morelli}

Formou-se em Tecnólogo em Processamento de Dados pela PUC-Rio, em 1990. Trabalha desde 1997 como administrador de bases de dados corporativas. Autor dos livros Oracle 8: SQL, PL/SQL e Administração (2000), SQL Server 2000 Fundamental (2001) e Oracle 9i Fundamental (2002), todos pela Editora Érica. Desde 1986 está envolvido com tarefas de treinamento, tendo ministrado cerca de 6.400 horas e desenvolvido mais de trinta apostilas. Também contribui para revistas do ramo tal como SQL Magazine.

Ficha Catalográfica

Morelli, Eduardo Maria Terra
Recriação automática de índices em
um SGDB relacional / Eduardo Maria Terra
Morelli ; orientador: Sérgio Lifschitz. - Rio de
Janeiro : PUC, Departamento de Informática,
2006. 120 f. : il. ; 30 cm
Dissertação (mestrado) - Pontifícia
Universidade Católica do Rio de Janeiro,
Departamento de Informática.

CDD: 004 
À Monick, Leonardo e Thiago, cúmplices em cada pequena conquista que viabilizou este grande trabalho. 


\section{Agradecimentos}

Ao longo do período no qual desenrolou-se esta dissertação, aconteceram inúmeros momentos de plena euforia e outros de completo desânimo. Felizmente, contei com precisas orientações do Professor Sergio Lifschitz, responsável direto pela manutenção dos trabalhos no rumo adequado. Os incontáveis encontros proporcionaram debates muito interessantes, fazendo as várias horas parecerem alguns minutos.

Devo agradecer a Marcos Salles pela sua excelente dissertação, que lançou as bases a partir das quais pude edificar este trabalho.

Obrigado a Anolan Milanés pelos inúmeros pedidos de socorro atendidos. Sua dissertação também contribuiu para determinar os rumos a seguir.

À amiga Janaína Oleinik, pelas horas a fio de estudos e pelo apoio constante.

Agradeço aos meus valorosos ajudantes, Felipe Rondon e Daniel Seabra, por valiosas contribuições de resultados experimentais.

Finalmente, não poderia esquecer de José Maria Monteiro, cujas dicas foram inestimáveis em diversos momentos. 


\section{Resumo}

Morelli, Eduardo Maria Terra; Lifschitz, Sérgio. Recriação Automática de Índices em um SGBD Relacional. Rio de Janeiro, 2006. 120p. Dissertação de Mestrado - Departamento de Informática, Pontifícia Universidade Católica do Rio de Janeiro.

Uma dentre as muitas tarefas desempenhadas por DBAs consiste em tentar garantir que os tempos de respostas dos comandos submetidos por usuários a um grande SGBDR não excedam valores previamente acordados. Esta dissertação segue uma linha de estudos denominada auto-sintonia de índices, que preconiza a realização de ajustes automáticos na execução de consultas SQL, visando reduzirlhes tempos de resposta, a partir de alterações no conjunto de índices: criação, eliminação e recriação. Este trabalho teve como ponto de partida a dissertação de Marcos Salles [32], que seguiu a mesma linha, propondo um mecanismo automático de criação de índices. Esta dissertação estende [32], primeiro submetendo sua implementação a uma carga de trabalho alternativa e depois realizando eliminações e reconstruções de índices automáticas, levando em consideração níveis de preenchimento de páginas alternativos. Também foram realizados testes utilizando ferramentas comerciais, Oracle 10g e SQL Server 2005, para avaliar quão eficaz comportou-se a implementação proposta em [32]. Vale ressaltar que os testes realizados limitaram-se à criação de índices, já que as ferramentas não oferecem facilidades de reconstrução automática. Diferentemente dos trabalhos publicados nessa linha de estudos e das ferramentas comerciais disponíveis, foi criado um protótipo que não se limita a sugerir novos índices; também são eliminados os que deixaram de ser interessantes, porém, antes ocorre uma avaliação para verificar se a reconstrução não seria mais adequada. Criou-se, inclusive, uma heurística rudimentar que avalia um índice a ser destruído e recomenda sua reconstrução, caso atenda a determinados requisitos.

\section{Palavras-chave}

Auto-sintonia; SGBD; Índices; PostgreSQL; Fragmentação 


\section{Abstract}

Morelli, Eduardo Maria Terra; Lifschitz, Sérgio. Automatic Reindexing in Relational Databases. Rio de Janeiro, 2006, 120p. MSc. Dissertation Departamento de Informática, Pontifícia Universidade Católica do Rio de Janeiro.

One of the most important tasks of Database Administrators certainly is to guarantee optimal response times to statements submitted by users of big RDBMS. Our dissertation deals with Index Self-tuning, which means creating, dropping or recreating indexes automatically, in order to decrease SQL queries durations. We start from Marcos Salles' dissertation [32], which proposed an automatic way of creating indexes. We extend [32] in many ways: first using a different workload, TPC-H like. Second, following created indexes inspecting its usage. Finally, we have gotten to drop and, mostly, recreate indexes using different fillfactor in leaf pages. Also, we have elaborated many tests using commercial tools, Microsoft SQL Server 2005 and Oracle 10g in order to ratify [32] ideas. Unfortunatelly, we could not test automatic dropping and recreating in these tools, as long as they do not offer this kind of functionalities. Unlike related work and commercial tools, we have created a code prototype that not only suggests new indexes creations, but also drops and recreates indexes using an own heuristics. To validate our ideas we have used a TPC-C like workload, but we had to make some changes to increase updates and force reindexing.

\section{Keywords}

Self-tuning; RDBMS; Indices; PostgreSQL; Fragmentation 


\section{Conteúdo}

1 Introdução 14

1.1. Contexto 14

1.2. O Problema 16

$\begin{array}{lll}1.3 & \text { Estrutura da Dissertação } & 18\end{array}$

2 Avaliação da Eficácia da Heurística de Benefícios com Cargas OLAP 20

2.1 Trabalhos Correlatos 20

2.2 Auto-sintonia Global de SGBDs 22

2.3 Criação Autonômica de Índices em Bancos de Dados 24

Heurística de Benefícios $\quad 24$

Arquitetura da Implementação 26

$\begin{array}{ll}\text { Resultados Obtidos } & 28\end{array}$

$\begin{array}{ll}2.4 \text { Agente de Benefícios e TPC-H } & 29\end{array}$

EVALUATE 30

Seletividade 32

Trabalhos Relacionados com Avaliações TPC-H 34

2.5 Resultados Experimentais com PostgreSQL 36

2.6 Resultados Experimentais com SQL Server 2005

2.7 Resultados Experimentais com Oracle 10g 40

2.8 Comentários Finais 41

3 Extensões à Heurística de Benefícios 43

3.1 Atribuição Cumulativa de Benefícios a Índices 43

3.2 Acompanhamento de Índices Criados: Bônus 44

$\begin{array}{ll}\text { Malus } & 47\end{array}$

3.3 Eliminação Automática de Índices $\quad 47$

3.4 Implementações: Bônus e Eliminação Automática de Índices 48

$\begin{array}{ll}\text { Camada Crença } & 48\end{array}$

$\begin{array}{ll}\text { Camada Raciocínio } & 50\end{array}$ 
Camada Ação 51

3.5 Resultados Experimentais 51

$\begin{array}{ll}\text { Ambiente } & 51\end{array}$

Estrutura dos Testes $\quad 51$

Conclusões $\quad 55$

3.6 Comentários Finais $\quad 55$

4 Reconstrução Automática de Índices 57

4.1 Bases da Heurística de Reconstrução Automática de Índices 57

$\begin{array}{ll}\text { Grau de Fragmentação } & 59\end{array}$

$\begin{array}{ll}\text { Tamanho } & 60\end{array}$

$\begin{array}{ll}\text { Varreduras } & 60\end{array}$

4.2 Fator de Preenchimento 61

4.3 GETSIZE $\quad 62$

4.4 Heurística de Reconstrução Automática de Índices 65

$\begin{array}{ll}4.5 \text { Estrutura Funcional } & 67\end{array}$

$\begin{array}{ll}\text { Camada Sensor } & 68\end{array}$

$\begin{array}{ll}\text { Camada Crença } & 69\end{array}$

$\begin{array}{ll}\text { Camada Raciocínio } & 70\end{array}$

Camada Ação $\quad 70$

$\begin{array}{ll}\text { Camada Colaboração } & 71\end{array}$

4.6 Interações entre Agentes $\quad 73$

4.7 Implementações e Resultados Obtidos 77

$\begin{array}{ll}\text { Estrutura dos Testes } & 78\end{array}$

4.8 Comentários Finais $\quad 80$

5 Conclusões e Trabalhos Futuros 82

Contribuições da Dissertação 83

Trabalhos Futuros $\quad 83$

$\begin{array}{lr}\text { Bibliografia } & 87\end{array}$

$\begin{array}{ll}\text { Apêndice A Benchmark TPCH } & 91\end{array}$

$\begin{array}{ll}\text { A.1 TPC } & 91\end{array}$ 
A.2 TPCH 92

A.3 Consultas Escolhidas $\quad 95$

$\begin{array}{ll}\text { Consulta } 1 & 96\end{array}$

$\begin{array}{lr}\text { Consulta } 2 & 97\end{array}$

$\begin{array}{ll}\text { Consulta } 4 & 98\end{array}$

Consulta $10 \quad 99$

$\begin{array}{ll}\text { Consulta } 17 & 100\end{array}$

A.3 Demais Consultas 102

Apêndice B Avaliação de Ferramentas de Apoio ao DBA 103

B.1 - Introdução 103

$\begin{array}{ll}\text { B.2 - BMC } & 105\end{array}$

B.3 - Embarcadero 112

B.4 - Idera 112

B.5 - Veritas 116 


\section{Lista de figuras}

Figura 1.1: consulta realizando cinco varreduras sobre uma tabela $\quad 17$

$\begin{array}{ll}\text { Figura 1.2: índice não fragmentado. } & 17\end{array}$

$\begin{array}{ll}\text { Figura 1.3: índice fragmentado } & 17\end{array}$

Figura 2.1: estratégia de Avaliação de consultas. 25

Figura 2.2: estratégia de Avaliação de atualizações 26

Figura 2.3: framework de construção de agentes de [KPP+99]. 27

Figura 2.4: exemplo de execução de evaluate, cujo argumento (select) tem sua execução inibida. 31

Figura 2.5: o otimizador proporciona ganhos decrescentes à medida que piora a seletividade 34

Figura 3.1: estrutura da tabela Venda $\quad 45$

Figura 3.2: comando executado sobre a tabela Venda 45

Figura 3.3: outro comando sobre a tabela Venda 45

Figura 3.4: estratégia de avaliação de consultas $\quad 50$

$\begin{array}{ll}\text { Figura 3.5: estratégia de avaliação de atualizações } & 50\end{array}$

Figura 4.1: nível folha de um índice recém criado. 58

Figura 4.2: nível folha de um índice que sofreu um page-split. 58

Figura 4.3: nível folha de um índice com alto grau de fragmentação. $\quad 59$

Figura 4.4: fórmula que obtém o grau de fragmentação de um índice 59

Figura 4.5: consulta efetuando várias varreduras sobre a tabela Venda. $\quad 60$

Figura 4.6: investigação do tamanho de uma tabela em PostgreSQL 62

Figura 4.7: comando fragmentando índice sobre o campo num. 62

Figura 4.8: novos valores obtidos em consulta à metabase, após fragmentar o índice. 63

Figura 4.9: exemplo de utilização da função pgstattable. 63

Figura 4.10: exemplo de utilização da função pgstatindex. 64

Figura 4.11: criação de um índice parcialmente preenchido 64

Figura 4.12: recriação de um índice utilizando fator de preenchimento maior 65

Figura 4.13: fórmula para obtenção do fator de preenchimento 66

Figura 4.14: diagrama de classes envolvidas na configuração das camadas $\quad 67$ 
Figura 4.15: diagrama de classes na camada Sensor $\quad 68$

Figura 4.16: diagrama de classes na camada Crença 69

Figura 4.17: diagrama de classes na camada Raciocínio $\quad 70$

Figura 4.18: diagrama de classes na camada Ação 71

Figura 4.19: diagrama de classes na camada Colaboração 72

Figura 4.20: interação entre agentes via camada Colaboração 73

Figura 4.21: Interação causada pelo serviço first_usage: primeira parte 75

Figura 4.22: Interação causada pelo serviço first_usage: segunda parte $\quad 76$

Figura A.1: esquema de Dados base de TPC-H 92

Figura A.2: totais consolidados para transações financeiras ocorridas em um determinado período 96

Figura A.3: seleciona qual fornecedor deve ser escolhido para realizar um pedido de um determinado componente em uma dada região 97

Figura A.4: verifica eficácia do sistema de controle de prioridades de pedidos e checa níveis de satisfação de clientes. 98

Figura A.5: mostra clientes que podem ter tido problemas com entregas. $\quad 99$

Figura A.6: determina a possível perda decorrente caso alguns pedidos tenham reduzidas pequenas quantidades de componentes. Isto permitiria reduzir o nível de overhead, permitindo maior concentração nas grandes compras. 100

Figura A.7: exibe descontos produzidos segundo diferentes estratégias utilizadas.

Figura A.8: nova versão para consulta 19.

Figura B.1: ambiente da ferramenta BMC SQL Explorer 106

$\begin{array}{ll}\text { Figura B.2: consulta típica acessando várias tabelas } & 107\end{array}$

$\begin{array}{ll}\text { Figura B.3: resultado de análise de comando SQL } & 108\end{array}$

Figura B.4: resultado de um Index Advisor. 109

Figura B.5: comandos em busca de índices hipotéticos 110

Figura B.6: comparação entre dois comandos: um com índice hipotético e outro sem.

Figura B.7: ambiente da ferramenta Idera SQL Diagnostic Manager 113

Figura B.8: identificando uma consulta agressiva 115

$\begin{array}{ll}\text { Figura B.9: Agent Manager } & 117\end{array}$

Figura B.10: ambiente da ferramenta InDepth 118 


\section{Lista de tabelas}

Tabela 2.1: 6 consultas escolhidas e 8 índices propostos pelo toolkit.

Tabela 2.2: 6 consultas escolhidas, 9 índices criados pelo agente e quantas vezes foi necessário executar com evaluate cada consulta para chegar às criações.

Tabela 2.3: 6 consultas escolhidas, 11 índices criados pelo SQL Server 2005. 39

Tabela 2.4: 6 consultas escolhidas, 10 índices criados pelo Oracle 10g.

Tabela 3.1: sequência de comandos revelando um caso típico de promoção de um índice hipotético em real.

Tabela 3.3: transações disparados pelo toolkit e respectivas quantidades de comandos

Tabela 3.4: transações alteradas com intuito de forçar a eliminação de índices 54

Tabela 4.1: relação de serviços caracterizando a cooperação entre agentes. 72

Tabela 4.2: valores de parâmetros relevantes de inicialização do PostgreSQL 77

Tabela 4.3: resumo da bateria de testes 80

Tabela A.1: testes constituindo o benchmark TPC-H 94

Tabela B.1: relação de fabricantes visitados. 103

Tabela B.2: relação de módulos da família SmartDBA do fabricante BMC 105

Tabela B.3: elementos da interface do produto BMC SQL Explorer 107

Tabela B.4: resumo de funcionalidades do produto BMC SQL Explorer 111

Tabela B.5: serviços oferecidos pela ferramenta SQL Diagnostic Manager 115

Tabela B.6: resumo de funcionalidades do produto Idera SQL Diagnostic $\begin{array}{ll}\text { Manager } & 116\end{array}$

Tabela B.7: principais elementos da ferramenta InDepth 118

Tabela B.8: resumo de funcionalidades do produto Veritas InDepth 119 